\title{
Hypochlorous Acid-Mediated Protein Oxidation: How Important Are Chloramine Transfer Reactions and Protein Tertiary Structure?
}

\section{SUPPLEMENTARY DATA}

\author{
David I. Pattison*, Clare L. Hawkins and Michael J. Davies
}

The Heart Research Institute, 114 Pyrmont Bridge Road, Camperdown, Sydney, NSW 2050, Australia.

Running Title: Predicting amino acid loss in HOCl-mediated protein damage

\footnotetext{
${ }^{\dagger}$ This work was supported by grants from the Australian Research Council under the ARC Centres of Excellence program, and the National Health and Medical Research Council.
}

* To whom correspondence should be addressed: Telephone: +61-2-8208-8900. Fax: +61-29565-5584. Email: pattisond@hri.org.au 
Table S1. The reactions and corresponding rate constants used to define the computational models for the reactions of $\mathrm{HOCl}$ with insulin and lysozyme. These kinetic parameters were used in conjunction with the composition data in Table 4.

\begin{tabular}{|c|c|c|}
\hline Reaction Number & Reaction & $\mathrm{k} / \mathbf{M}^{-1} \mathbf{s}^{-1 a}$ \\
\hline & $\underline{\mathrm{HOCl}+}$ & \\
\hline 1 & Arg $\rightarrow$ Arg-Cl & 26 \\
\hline 2 & Asn $\rightarrow$ Asn-Cl & 0.03 \\
\hline 3 & Cys $\rightarrow$ Cys-Cl & $3.0 \times 10^{7}$ \\
\hline 4 & Cystine $\rightarrow$ Cystine-Cl & $1.6 \times 10^{5}$ \\
\hline 5 & $\mathrm{Gln} \rightarrow \mathrm{Gln}-\mathrm{Cl}$ & 0.03 \\
\hline 6 & His $\rightarrow$ His-Cl & $1.0 \times 10^{5}$ \\
\hline 7 & Lys $\rightarrow$ Lys-Cl & $5.0 \times 10^{3}$ \\
\hline 8 & Met $\rightarrow \operatorname{MetS}(\mathrm{O})$ & $3.8 \times 10^{7}$ \\
\hline 9 & Trp $\rightarrow$ Trp-Cl & $1.1 \times 10^{4}$ \\
\hline 10 & Tyr $\rightarrow$ Cl-Tyr & 44 \\
\hline 11 & $\alpha$-amino $\rightarrow \alpha$-amino-Cl & $1.0 \times 10^{5}$ \\
\hline 12 & Backbone amide $\rightarrow$ backbone-Cl & 10 \\
\hline \multirow[t]{2}{*}{13} & MetS(O) $\rightarrow$ Met sulfone & 155 \\
\hline & $\underline{\text { His- }} \mathrm{Cl}+$ & \\
\hline 14 & Arg $\rightarrow$ Arg- $\mathrm{Cl}+$ His & 1.3 \\
\hline 15 & Cys $\rightarrow$ Cys-Cl + His & $5.0 \times 10^{6}$ \\
\hline 16 & Cystine $\rightarrow$ Cystine-Cl + His & $2.1 \times 10^{4}$ \\
\hline 17 & Lys $\rightarrow$ Lys-Cl + His & 200 \\
\hline 18 & Met $\rightarrow$ MetS(O) + His & $1.6 \times 10^{6}$ \\
\hline 19 & Trp $\rightarrow$ Trp-Cl + His & $2.1 \times 10^{3}$ \\
\hline
\end{tabular}


Lys-Cl+

\section{$\underline{\alpha \text {-amino-Cl }+}$}

\section{Chloramine decomposition}

${ }^{a}$ All rate constants are apparent second-order rate constants at pH $7.0-7.5$ and $20-25^{\circ} \mathrm{C}$ except where otherwise stated (from current paper, $(11,33,35,36)$ and reviewed in (18)). ${ }^{b}$ First-order rate constants $\left(\mathrm{k} / \mathrm{s}^{-1}\right)$ derived for chloramine decomposition at $\mathrm{pH} 7.4$ and $22{ }^{\circ} \mathrm{C}$. 